\title{
LES COLLECTIONS D'OEUVRES D'ART DU METAL EMAILLE HISPANO-MUSULMAN DANS LES MUSEES MONDIAUX HORS D'ESPAGNE
}

Por

VALÉRIE GONZALEZ

La réputation des Musulmans d'Espagne en matière d'art somptuaire n'est certes plus à faire, mais il n'en demeure pas moins utile de dénicher les exemples concrets qui l'attestent. II est en particulier une technique d'art du métal savante, l'incrustation d'émaux polychromes, dans laquelle les ateliers auliques excellèrent et qui permit aux exigeants souverains d'Al-Andalus de se constituer de fabuleuses panoplies aussi riches que raffinées (1). De cette production islamique ibérique originale subsiste un échantillonage conséquent qui compte de véritables chef-d'oeuvres. La pièce la plus célèbre et la plus représentative, en raison de sa qualité artistique remarquable et du mythe qui auréole son ancien possesseur et commanditaire, est l'épée du dernier Sultan de Grenade, Boabdil, conservée au Musée de l'Armée de Madrid. L'Espagne possède un certain nombre de ces objets émaillés musulmans, disséminés dans les divers lieux de conservation de son territoire, tels que les bijoux et éléments de harnachement de cheval du Musée National Archéologique, de l'Institut Valencia de Don Juan et du Musée Lazaro Galdiano de Madrid, où l'épée mauresque de la Réal Arméria de la même ville et celle du Musée San Telmo de San Sebastian. Mais force est de constater que les collections espagnoles ne peuvent à elles seules donner l'ampleur -qui fut considérable- de l'art d'émailler sur métal en Andalus. En effet, une prospection scrupuleuse dans différents musées extérieurs à la péninsule ibérique révéla de somptueux ornements masculins et féminis, de valeur artistique au moins égale à celle de l'épée du Sultan (citée ci-dessus) pour certains,

(1) Conférer: V. GONZÁLEZ: «L'émaillerie cloisonnée dans Al-Andalus», in Revue de /'Occident Musulman et de la Méditerranée, Aix-en-Provence, n. ${ }^{\circ} 40,1985$, p. 55-73. 
et mit en lumière la réelle portée historique de la technique dans l'histoire des arts industriels hispano-musulmans (2).

Ainsi, le matériel archéologique le plus ancien qui prouve la pratique de l'émaillerie dès les débuts de l'existence de l'Etat musulman en Espagne, se trouve aux Etats-Unis d'Amérique. Cet état de fait valut que, communément, l'on occulta une grande partie de l'histoire de cet art pour ne s'intéresser qu'à sa phase ultime, correspondant au règne nasride (3).

\section{Les emaux du Califat de Cordoue (316-317/929-421-422/1030). Walters Art Gallery, Baltimore}

De beaux émaux cloisonnés sur or attestent que l'émaillerie s'est introduite et développée dans la métallurgie d'Al-Andalus au moins dès la restauration syrienne omeyyade. Ils sont conservés à la Walters Art gallery de Baltimore, sous le numéro d'inventaire 57.1596 (1-45). Ils font partie d'un lot de 45 élémets de bijoux d'or qui proviendraient, selon le musée, de Madinat al-Zahrā, et qui dateraient du 10ème siècle. Ils consistent en une petite étoile, de deux centimètres environ, et un croissant de lune, de taille à peu près similaire. Sur la première, l'émail apparaît sous forme d'une pastille rapportée au centre, ornée d'un motif d'oiseau; sur le second, il recouvre toute la plaque et était lui-même destiné à être inséré sur une plaque plus grande, vraisemblablement sur une des boucles d'oreille discoïdales du même lot, flanquée d'un croissant de lune central évidé. Ces divers éléments soit entraient dans des parures, soit étaient cousus au tissu de riches vêtements d'apparat. La facture de l'émail, extrêmement fine, minutieuse et délicate, signale un art consonmmé. Ainsi, les Omeyyades d'Espagne, avec les Fatimides d'Egypte qui fournirent une émaillerie comparable à celle de ces derniers, apparaissent, d'après les témoignages artistiques conservés, comme les premiers producteurs d'émaux sur métal du monde arabomusulman au Moyen-Age.

II ne subsiste pas de matériel sûrement daté de l'époque suivante des règnes des Taifas, indiquant la continuité de la technique après la chute du Califat cordouan. Cependant, une paire de boucles d'oreille émaillées musulmanes, de provenance inconnue, pourrait bien s'intégrer à l'art des hispano-omeyyades. Le seul indice possible permettant de suivre, peut-être, l'évolution de l'émaillerie en Andalus sans interruption sous les Taifas, ne se trouve pas non plus dans un musée espagnol, mais au Métropolitan Museum de New-York.

\section{Boucles d'oreille émaillées musuimanes-Espagne? Metropolitan Museum of Art, New-York}

Ces belles boucles d'oreille émaillées cloisonnées sur or, sont situées, sous ré-

(2) Ce travail fut effectué par moi-même et se trouve développé et argumenté dans ma thèse: «Origine, développe ment et diffusion de l'émaillerie sur métal en Occident Musulman", Université de Provence 1, Mai 1989, 2 vol., 712 pages.

(3) Deux historiens seulement parlent de cette ancienne production sans que leurs affirmations n'aient trouvé d'échos auprès des auteurs intéressés de près ou de loin par la question: CHAUNCEY-ROSS (M): «An Egypto-arabic cloisonné enamel" in Ars Islamica, n. ${ }^{\circ} 7,1940$, p. 165-167; HILDBURGH (W.L.): Medieval spanish enamels, Oxford, 1936. 
serves toutefois, au Xième siècle par M. JENKINS (4). Elle souligne le rapprochement technique et stylistique avec des boucles fatimides de même époque, mais ne parle pas de celles d'Espagne omeyyade qui leurs ressemblent également. II s'agit en fait d'un prototype de parure discoidale, pourvu d'un décor émaillé rapporté en forme de croissant de lune. Il est représenté dans le lot de Madinat al-Zahrā de Baltimore (mentionné plus haut) et plus abondamment dans le matériel fatimide. A regarder de près, tout en faisant montre d'une indéniable originalité laissant planer le doute, ces boucles du Métropolitan Museum offrent maints traits communs avec les émaux andalous : la palette ainsi que les motifs en forme de coeur à palmette étoilée à l'intérieur du croissant, semblent s'y référer. La composition graphique en particulier, complexe, ne rencontre aucune similitude stylistique en dehors du cadre artistique de l'Andalousie musulmane (5).

Après les règnes de Taifas, le créneau temporel des empires hispano-berbères s'avère être pareillement comblé par un unique témoin dont la datation est fiable: une somptueuse paire de boucles d'oreille du 12ème siècle, exposée au Musée National du Koweit.

Boucles d'oreille émaillées d'Espagne almohade (6ème, 7ème siècles/12ème, 13 ème siècles). Musée National, Koweit

La technique d'émaillage utilisée pour l'ornementation de ce joyau est la même que pour les parures précédentes, le cloisonné sur or; en revanche, la morphologie et le répertoire graphique diffèrent. Le thème des émaux procède de l'écriture arabe cursive qui effectivement apparaît en Andalus au 12ème siècle. Les caractères denses et fortement ondulés, formulent la sourate CXII du Coran proclamant l'unicité de Dieu (6). Un cadre géométrique rigide, qui est la structure même de la boucle, emprisonne ce décor calligraphié agité et foisonnant, dans le pur style almohade, à la manière par exemple du décor des monnaies de la même période.

L'Espagne possède un élément de bronze que l'on pourrait éventuellement ranger dans la même époque. Il s'agit d'un petit passe-courroie garni d'émail champlevé, d'usage courant, dont la qualité n'a cepedant rien de comparable avec les bijoux ci-dessus. En revanche, les collections espagnoles deviennent bien plus conséquentes en ce qui concerne l'émaillerie du Royaume de Grenade consécutif à la débacle des Almohades dans la péninsule. Pourtant, bien des chefs-d'oeuvres de l'émaillerie andalouse nasride ne peuvent être admirés en Espagne mais à l'étranger. C'est pourquoi un recensement sérieux s'impose pour restituer sa véritable envergure à cet art tardif.

(4) M. JENKINS donne un petit commentaire sur ces bijoux dans le catalogue d'une exposition qui eut lieu au Métropolitan Museum of Art: JENKINS (M) et KEENE (M): Islamic Jewelry in the Metropolitan Museum of Ant, New-York, M.M.A., 1983, p. 76.

(5) Dans le présent article, le propos n'est pas d'argumenter longuement sur l'esthétique des objets cités. Signalons que ces remarques peuvent être par ailleurs amplement développées et étoffees.

(6) Les boucles d'oreille du musée du Koweit sont magnifiquement reproduites dans le catalogue de l'exposition sur les trésors de l'Islam au Musée Rath de Genève: Trésors de I'Islam, Genève, Musée rath, 1985, p. 344. 
Les emaux du royaume de Grenade (628-629-897-898/1231-1492)

Metropolitan Museum of Art, New-York ; Walters Art Gallery, Baltimore;

British Museum et Victoria and Albert Museum, Londres ; Kungstgammlungen

Museen, Kassel ; Musée du Louvre et Cabinet des Medailles, Paris

Les musées hors d'Espagne cités dans le titre ci-dessus, renferment tous des pièces uniques pour leur qualité artistique remarquable et certaines pour leur rareté. A ce titre, le Metropolitan Museum est le plus riche. Dans le domaine de la bijouterie, ses vitrines et ses réserves comptent une ceinture complète en velours et cuivre cloisonné, et des colliers émaillés sur or filigrané d'un raffinement nettement supérieur à celui des parures de même type exhibées au Musée National Archéologique de Madrid. Parmi ces bijoux du musée américain, se trouve notamment un pectoral, le plus sophistiqué et connu du genre classique grenadin à lourds pendentifs et à bobines (qannuta). En outre, il contient à lui seul un concentré des données esthétiques de l'art de la parure nasride, avec ses différents aspects iconographiques et ses contradictions plastiques. En matière d'équipement militaire, le Metropolitan Museum possède une très belle paire d'étriers couverts d'émaux champlevés, des médailles de harnachement de cheval et une épée mauresque émaillée, fortement remaniée, qui ne rivalise pas en perfection avec la fameuse arme de Boabdil du Musée de l'Armée de Madrid. Mais le clou de sa collection est une pièce exceptionnelle dont aucun autre musée du monde ne donne à voir l'équivalent. Il s'agit d'un casque de type européen dit «salade», en acier doré à la feuille, incrusté sur toute la surface d'émaux cloisonnés sur argent d'une très grande finesse, en forme de pastilles, gouttes et cartouches alternés, et rehaussé par endroit d'écritures arabes niellées. Cet objet, à la fois magnifique et insolite, constitue une démonstration fort significative de l'art de l'armement d'apparat à l'époque du Royaume Grenadin. Notamment, le mélange des sources européennes chrétiennes et musulmanes y est clairement lisible.

C'est un talisman émaillé cloisonné, en forme de boîte contenant des feuillets couverts d'inscriptions coraniques, qui fait la richesse du matériel grenadin de la Walters Art Gallery de Baltimore. Il est le seul bijou prophylactique nasride connu monté de la sorte, à la façon des cassolettes de colliers préservatifs du Maghreb contemporain. Un très beau pommeau d'épée, flanqué d'un disque orné d'émaux cloisonnés, fait également partie de ce matériel.

Les pièces des deux musées londoniens ne sont pas moins dignes d'intérêt. Avec celle du Metropolitan Museum, la ceinture émaillée du British Museum, à peu près semblable au niveau de la structure et des décors, compose tout l'échantillon subsistant de la production grenadine de ce type d'ornement. A priori, il n'en existe pas, dans leur intégralité, en Espagne. Heureusement, ces deux exemplaires subsistent pour nous montrer que cet élément particulier de la parure des Nasrides faisait autant l'objet d'un soin et d'une recherche artistiques que les joyaux pour les femmes, et les armes pour les hommes. Quant au Victoria and Albert Museum, on peut y admirer de superbes cloisonnés sur or formant d'élégants dessins sur une frette d'épée mauresque, disparue, qui devait être aussi richement ornée que celle de Boabdil.

Le musée de Kassel s'illustre également par une admirable épée de la même série, la plus belle avec cette dernière, suivie de très près par une autre conservée au Cabinet des Médailles de Paris. 

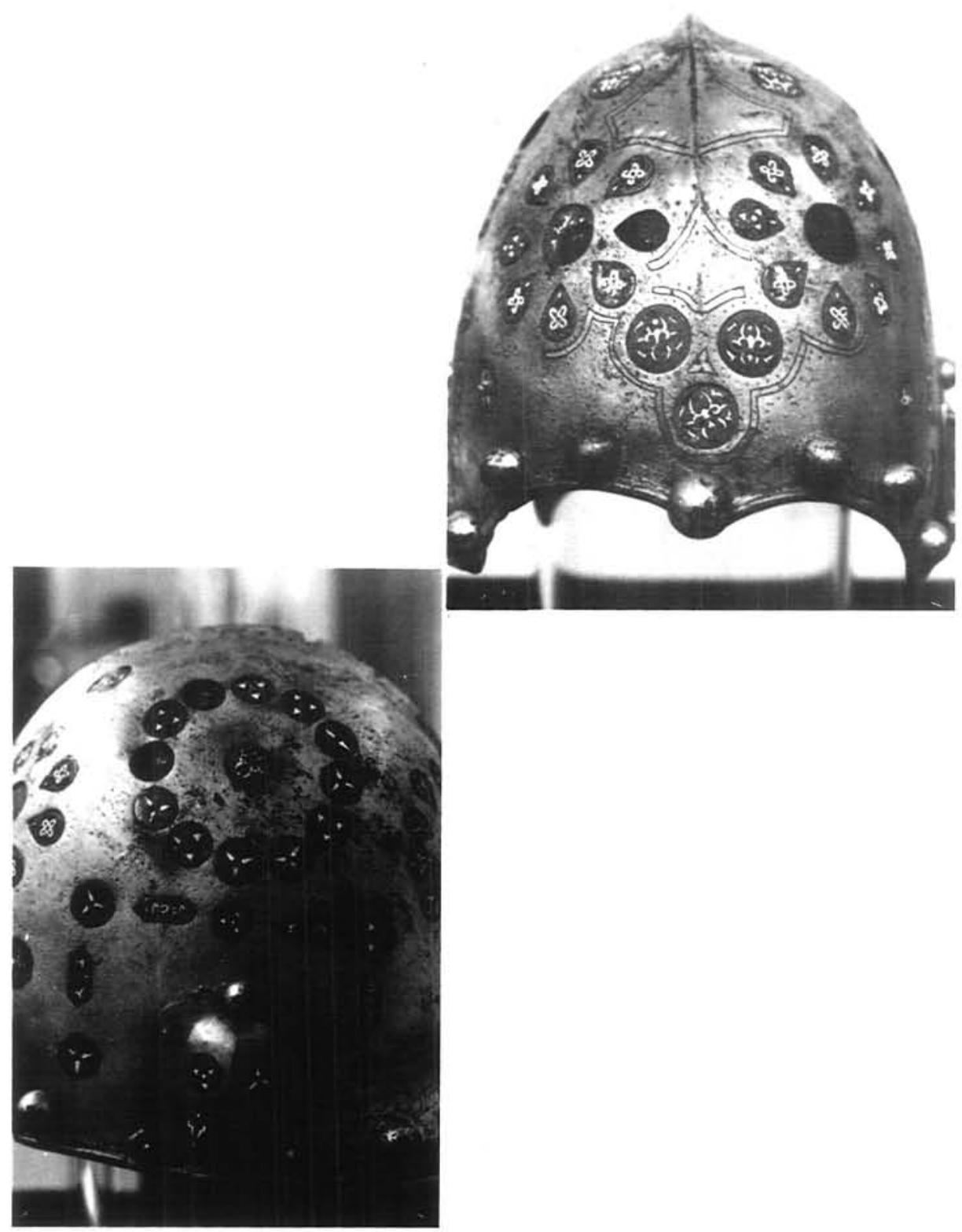

Casque nasride - Émail cloisonné sur or et argent plaqués sur acier - $15^{e}$ siècle - Grenade. METROPOLITAN MUSEUM OF ART - NEW YORK. Don de la «Vicent Astor Foundation». N. ${ }^{\circ}$ : d'inventaire 1983413. Clichés de V. GONZÁLEZ. 


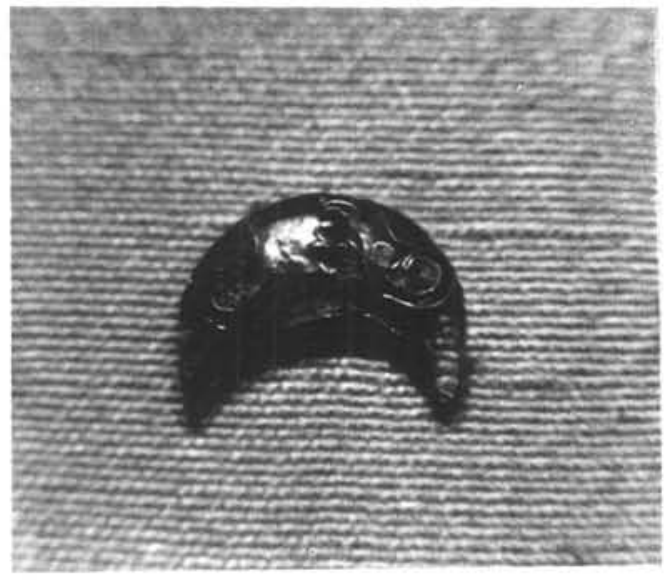

Élements d'un lot de bijoux hispanoomeyyades. Émail cloisonné sur or. $10^{e}$ siècle. Madınat al-Zahra (Cordue). WALTERS ART GALLERY OF BALTIMORE (USA). N. ${ }^{\circ}$ : d'inventaire: 571596 Clichés V. GONZÁLEZ.

A: croisant de lune, dimensions: $1 \mathrm{~cm}$. environ.

$\mathrm{B}_{1}$ et $\mathrm{B}_{2}$ : étoile et médaillon central circulaire émaillé, dimensions: $2 \mathrm{~cm}$. environ.
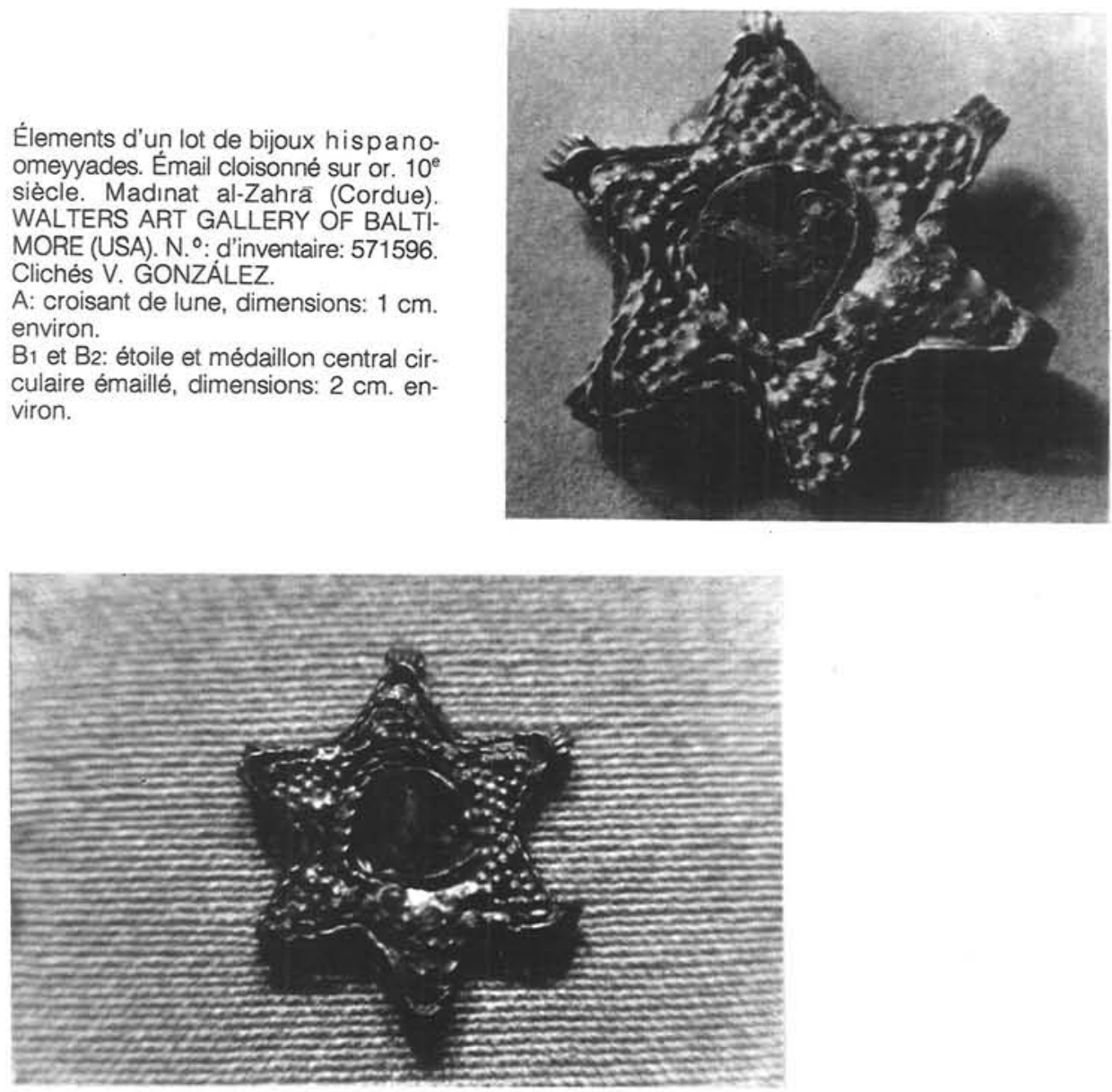

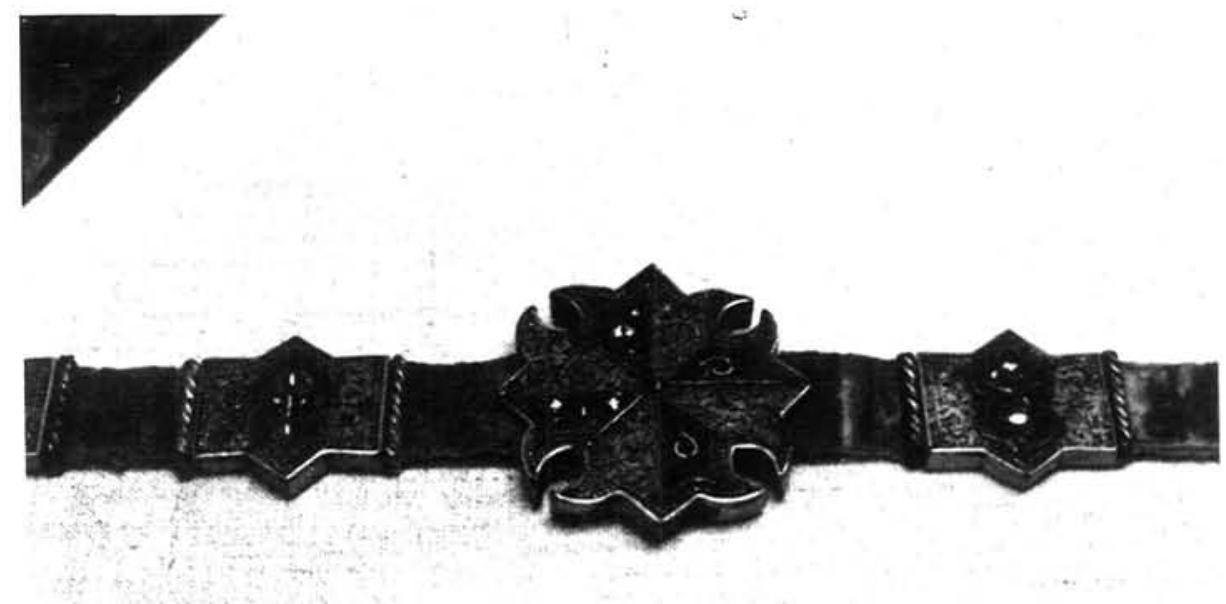

Ceinturon nasride. Émail cloisonné sur bronze doré et velours- $15^{e}$ siècle. Grenade. METROPOLITAN MUSEUM OF ART - NEW YORK. N. ${ }^{\circ}$ : d'inventaire: 17190962. Cliché V. GONZÁLEZ.

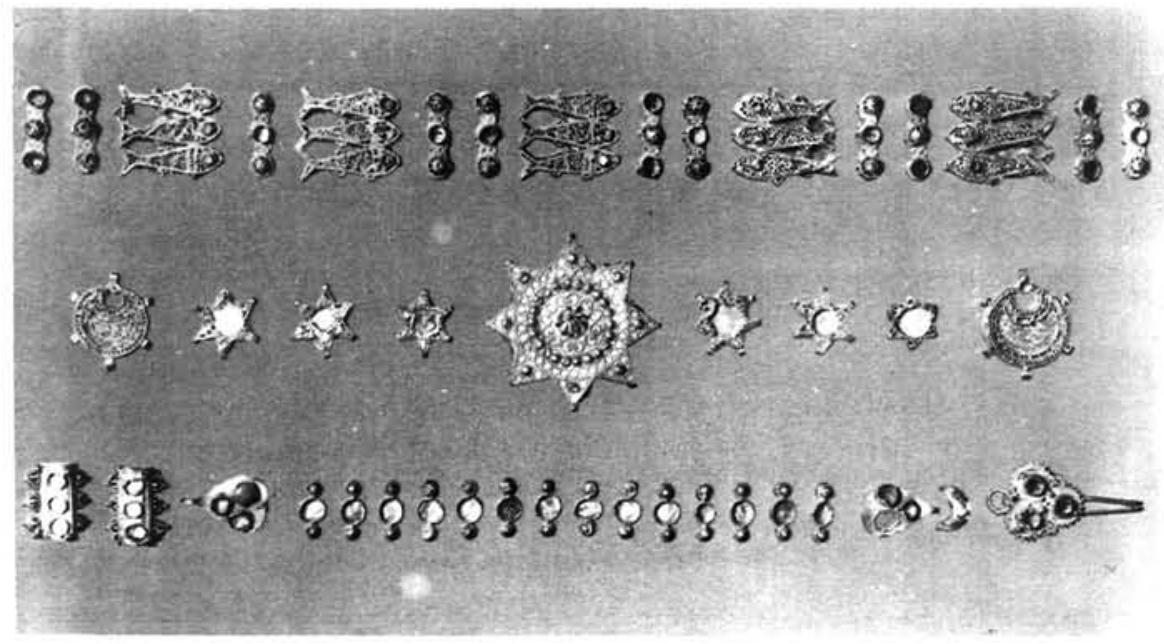

Lot de byjoux hispano-omeyyades. Or et émail cloisonné et gemmes. Madınât al-Zahra (Cordue). $10^{\circ}$ siècle. WALTERS ART GALLERY OF BALTIMORE (USA). N. ${ }^{\circ}$ : d'inventaire: 571596 . (45 éléments). 
Enfin, citons une amulette d'argent en forme de reliure de Coran, «kitāb», conservée au Musée du Louvre. Ce type d'objet sacré est très peu représenté puisque, en dehors de cette amulette, il ne reste que le talisman de la Walters Art Gallery.

Nous constatons que le panorama de l'emaillerie sur métal en Andalus serait bien incomplet si nous devions nous en tenir à ce que l'Espagne seule comporte dans ses lieux de conservation et, certes, il le demeure en raison des collections particulières inaccesibles ou dispersées avec le temps, et des secrets de musées inviolés. Considérons plutôt ce travail toujours inachevé, à la merci d'une surprise, jetant un peu plus de lumière sur la question sans la faire tout à fait. 